\title{
Design of Special-shaped Interchange between Expressway and Urban Road in Mountainous Cities
}

\author{
Shang Ye* \\ China Merchants Chongqing Communications Technology Research \& Design Institute CO., LTD. Chongqing 400067, \\ China
}

*Corresponding author: Shang Ye, 317540110@qq.com

\begin{abstract}
Driven by the rapid economic development, the development of transportation in China has begun to move towards mountainous areas. The climate environment, topography and landform of mountainous cities are different from those of plain areas. In mountainous cities, the area of opposite interchanges between expressways and urban roads is generally large, which has a certain contradiction with the topographic conditions of mountainous cities. Therefore, it is necessary to reasonably design the opposite interchanges between expressways and urban roads in mountainous cities. The author explores and analyzes the factors restricting the special-shaped interchange between expressway and urban road in mountainous cities and the main forms of special-shaped interchange, and puts forward a reasonable design scheme, hoping to make a smooth development of the special-shaped interchange in mountainous cities.
\end{abstract}

Keywords: Mountainous cities; Expressway; Urban Road; Special-shaped; Interchange

Publication date: September 2021; Online publication: September 30, 2021

\section{Introduction}

The special-shaped interchange between expressway and urban road is usually located around the urban area of mountainous cities, and the environmental conditions in mountainous areas are generally complex, which requires that the design work must be done in advance when building the special-shaped interchange between expressway and urban road in mountainous cities. In the process of specific design, the primary task is to analyze the main characteristics of special-shaped interchanges in mountainous urban expressways and urban roads. Secondly, before specific design, it is also necessary to accurately understand the main factors restricting the construction of special-shaped interchanges. At the same time, we also need to understand the main forms of special-shaped interchange, so as to better complete the design work.

2. Factors restricting special-shaped interchange between expressway and urban road in Mountainous Cities

In the design of special-shaped interchange between expressway and urban road in mountainous cities, there are many factors that restrict the implementation of the design scheme of special-shaped interchange.

\subsection{Limit of height difference}

One of the most obvious characteristics of mountainous cities is that the terrain fluctuates greatly and frequently, the breaking height is low, and there is a large height difference. In the selection of expressway line, the way of line expansion can be used to avoid restricting the implementation of special-shaped interactive interchange design scheme due to large height difference. In the specific design of specialshaped interchange between urban expressway and urban road in mountainous areas, if there is a large height difference between expressway, urban road main line and intersection line or continuous line, it is 
necessary to use spiral line development and back line development, natural line development and other ways to meet the requirements of the longitudinal slope of the ramp in the special-shaped interchange.

\subsection{Plane limit}

It is easy to be restricted by the plane when designing the special-shaped interchange between the expressway and the urban road in mountain city. That is, it is easy to be limited by obstacles when choosing the main section of urban expressway and urban road in mountainous areas. At this time, you can choose to remove them directly or avoid the restrictions of obstacles through the setting of bridges and tunnels. It is precisely because the special-shaped interchange between expressway and urban road in mountainous cities is limited by the land, so the development needs of the city should be considered in the design.

\section{Main forms of special-shaped interchange}

\subsection{A deformable interchange where ramps are separated from each other}

The land area of ordinary interchange is generally large, and the terrain of mountainous cities is usually complex, so it is difficult to meet the land demand of ordinary interchange construction ${ }^{[1]}$. If the interchange ramps are separated, although the total floor area may increase from the overall point of view, the floor area will decrease from the local point of view. Therefore, when designing urban highways and urban roads in mountainous areas, the interchange ramp can be divided into two parts by using the open space between two smaller mountains, which can not only completely preserve the original landform of mountainous cities, but also reduce the damage to mountains.

\subsection{A deformable interchange where main lines are separated from each other}

The deformation interchange with the main line separated from each other is a special-shaped interchange form in which the left and right lines of urban expressway and urban road in mountainous areas are distributed on both sides of the coast or mountain bag, and intersect it with another main line through the adoption of separated subgrade.

\section{Reasonable design scheme of special-shaped interchange between expressway and urban road in mountainous cities}

4.1. Design of separation ramp special-shaped interchange for expressway and city road in mountain city

The main reason for the ramp separation of Expressway and urban road conventional interchanges in mountainous cities is that there are obstacles in the setting area of interchanges, which limits the setting of conventional interchanges. For example, there are obstacles when setting ramps in sections such as mountain mouth, raised Highlands, ponds and natural pits ${ }^{[2]}$. Although the layout of conventional overpasses can also be carried out technically, long-span bridges, large-scale filling and excavation or demolition are usually required at that time, which will cause damage to the surrounding environment. Obviously, this is inconsistent with the needs of urban development, which is not conducive to ecological environmental protection, but also to improving the economy of project construction. Therefore, when designing the special-shaped interchange between expressway and urban road in mountainous cities in these sections, we must take into account the needs of urban development, and the applicability of interchange can be improved by means of ramp separation.

When arranging expressway lines in mountainous areas, it is necessary to conduct comprehensive analysis and consideration from the perspectives of preventing massive excavation of mountains, reducing the emergence of bridge and tunnel structures, protecting the surrounding ecological environment, reducing the difficulty of expressway construction and improving expressway benefits. The line location of 
Expressway in mountainous area is usually more suitable to be arranged at the foot of the hillside. For mountainous areas, the terrain connecting with the foot of the hillside is diverse, mainly including river banks, valleys, flat lands, etc., and there may be a prominent terrain at the connection, which is usually called "mountain mouth." Mountain mouth is a terrain often faced by mountainous urban expressway in route selection. When designing special-shaped interchange at this terrain, comprehensive analysis should be carried out from the aspects of construction technology and project cost control. Usually, short tunnels can be used to ensure the rapidity and environmental protection of expressways.

\subsection{Design of connect line development special-shaped interchange between urban expressway and urban road in mountainous area}

For mountainous urban expressways and urban roads, the connecting line is the collection of turning off ramps of expressways and urban roads, which can overcome all the problems faced by turning ramps. If there are obstacles in the ramp, it can be bypassed or partially expanded. If there are no obstacles and restrictions on the ramp, the connecting line can be set in the right turn quadrant with relatively large traffic flow, as long as the length of the connecting line meets the setting of toll stations and the demand of main lines for deceleration through the ramp ${ }^{[3]}$. However, the environment in mountainous areas is very complex. When designing special-shaped interchange, the requirements for the land scope in the plane and the height difference between roads are also strict. And high-speed difference and plane limitation usually exist together. At this time, when designing the special-shaped interchange bus for the expansion of urban expressway and urban road connecting lines in mountainous areas, upside down, spiral, turn back and natural expansion can be adopted, which can not only overcome the limitation of height difference, but also break through the plane limitation by means of line expansion and avoidance. Of course, for different wire spreading methods, they have their own advantages and disadvantages, and the applicable conditions are also different. Therefore, when selecting the line expansion mode, it can be selected from the perspectives of traffic capacity, driving speed, driver operation ability, project cost, construction difficulty, urban development demand and topographic conditions of urban expressways and urban roads in mountainous areas.

\subsection{Design of right turn ramp special-shaped interchange between urban expressway and urban road in mountainous area}

If you want to realize the smooth operation of right turn special-shaped ramps of urban expressways and urban roads in mountainous areas, you need to cross up or down with the main line twice. On one hand, the smooth operation of the right turn ramp is realized through continuous 270 degree left turn by using the confluence or diversion with the semi directional left turn ramp ${ }^{[4]}$. The route is usually located in the middle of the ring ramp and semi directional ramp, so the construction technology, operation capacity and operation speed are also between the ring ramp and semi directional ramp. Compared with the forms of directly connected right turn ramps, the detour length of right turn special-shaped ramps is significantly longer, various parameters and indicators are reduced, and the adaptability of ring ramps and turning traffic volume is basically the same, but it can complete merging with the main line in advance or diversion in a later step. On the other hand, use the ring ramp to merge or divert the right turn special-shaped ramp, and then complete the right turn through the crossing of the main line. Because this form of right turn ramp is realized by means of ring ramp, the driver needs to pass through a section of S-shaped Road, which requires high operation level of the driver, and the running speed will be limited. Therefore, this design method is only applicable to the case of obstacles on the right turn ramp. 


\section{Conclusion}

To sum up, when optimizing the design of mountainous urban expressway and urban road special-shaped interchange, we must comprehensively consider and analyze various factors restricting the construction of special-shaped interchange, and understand the main forms of special-shaped interchange. Thus, to better optimize the ramp separation of mountainous urban expressway and urban road, the display of connecting line, right turn ramp special-shaped interchange design, so that it can provide more high-quality expressway and urban road traffic services for the masses.

\section{Disclosure statement}

The author declares no conflict of interest.

\section{References}

[1] Wang CP, 2021, Importance of Comparison and Selection of Design Schemes for Key Node Sections of Urban Roads in Mountainous Areas. Northern Architecture, 6(04): 33-36.

[2] Fu CL, 2020, Discussion on Problems and Countermeasures in Road Engineering Design of Mountainous Cities. Communications Science and Technology Heilongjiang, 43(05): 43-44.

[3] Su XF, 2019, Analysis and Application of Key Points for Comparison and Selection of Urban Road Design Schemes in Mountainous Areas. Construction \& Design for Project, (17): 97-99.

[4] Gong L, 2018, Study on Route Selection of Urban Roads in Mountainous Areas -- Taking Tongxin Avenue in Bijie as an Example. Theoretical Research in Urban Construction (Electronic Version), (30): 6 . 